\title{
THE CONVEX HULL OF THE FINITE BLASCHKE PRODUCTS ${ }^{1}$
}

\author{
BY STEPHEN FISHER
}

\section{Communicated by John Wermer, June 28, 1968}

Let $U$ be the unit disc in the complex plane, $U=\{z|| z \mid<1\}$. A finite Blaschke product is an analytic function on $U$ which is either a constant of modulus one or of the form

$$
\lambda \prod_{i=1}^{N} \frac{z-\alpha_{i}}{1-\bar{\alpha}_{i} z}, \quad N=1,2, \cdots
$$

where $\lambda$ is a complex number of modulus one and $\alpha_{i} \in U$ for $1 \leqq i \leqq N$. It follows from [1, p. 12] that the set of functions analytic on $U$ and continuous on $\bar{U}$, the closure of $U$, which have modulus one on the unit circle consists precisely of the finite Blaschke products. The purpose of this note is to prove the following theorem, originally raised as a question by Phelps in [2].

THEOREM. Let $f$ be analytic on $U$, continuous on $\bar{U}$, and bounded by one. Then $f$ may be uniformly approximated on $\bar{U}$ by convex combinations of finite Blaschke products.

PRoof. For $0 \leqq t \leqq 1$ let $f_{t}(z)=f(t z)$ for $z \in U$. Since $f$ is continuous on $\bar{U}, f_{t}$ converges uniformly to $f$ as $t \rightarrow 1$. By a theorem of Caratheodory $\left[1\right.$, p. 13] there is a sequence $\left\{\Phi_{j}\right\}$ of finite Blaschke products of the form $(*)$ such that $\Phi_{j} \rightarrow f$ uniformly on compact subsets of $U$. It follows that given $\epsilon>0$ and $t<1$ we may find a finite Blaschke product $\Phi$ of the form (*) with $\left\|f_{t}-\Phi_{t}\right\|<\epsilon / 2$. Thus given $\epsilon>0$ there is a $t<1$ and a finite Blaschke product $\Phi$ of the form $(*)$ with $\left\|f-\Phi_{t}\right\|_{\infty}<\epsilon$. We now show that $\Phi_{t}$ is itself actually a convex combination of finite Blaschke products. It suffices to do this when $\Phi(z)=(z-\alpha)(1-\bar{\alpha} z)^{-1}$ since $(g h)_{t}=g_{t} h_{t}$ for any $g$ and $h$ and since the set of convex combinations of finite Blaschke products is clearly closed under multiplication. However, for $\alpha=r e^{i \theta}$ and $0 \leqq t \leqq 1$ we have

$$
\begin{aligned}
\frac{t z-\alpha}{1-\bar{\alpha} t z}= & \frac{t\left(1-r^{2}\right)}{1-r^{2} t^{2}}\left\{\frac{z-\alpha t}{1-\bar{\alpha} t z}\right\}+\frac{r\left(1-t^{2}\right)}{1-r^{2} t^{2}}\left\{-e^{i \theta}\right\} \\
& +\frac{(1-t)(1-r)}{1+r t}\{0\} .
\end{aligned}
$$

${ }^{1}$ Research partially supported by NSF Grant GP 8660. 
Since the factors in the first two sets of brackets are finite Blaschke products and the zero in the third is a convex combination of such, and since the coefficients are nonnegative and sum to 1 , the proof is complete.

\section{REFERENCES}

1. C. Caratheodory, Theory of functions. Vol. 2, Chelsea, New York, 1954.

2. R. R. Phelps, Extreme points in functions algebras, Duke Math. J. 32 (1965), 267-278.

Massachusetts Institute of Technology

\section{EXACTNESS OF INVERSE LIMITS}

\section{BY ULRICH OBERST}

Communicated by Saunders Mac Lane, May 20, 1968

I. The problem of this investigation is to characterize those small categories $X$ for which the inverse limit

$$
\operatorname{proj} \lim _{X}: A B^{X} \rightarrow A B
$$

is exact. Here $A B$ is the category of abelian groups, and $A B^{\boldsymbol{x}}$ is the category of functors from $X$ to $A B$. In this context I conjecture the following

TheOREM I. Let $X$ be a small category. Then the following assertions are equivalent:

(1) The inverse limit proj $\lim _{X}: A B^{\mathbf{x}} \rightarrow A B$ is exact.

(2) For every abelian category I with exact direct products, the inver se limit $\operatorname{proj} \lim _{X}: \mathfrak{A}^{\mathbb{X}} \rightarrow \mathfrak{A}$ is exact.

(3) Every connected component $Y$ of $X$ contains an object $y$ together with an endomorphism $e \in Y(y, y)$ such that the following properties are satisfied:

(i) $y$ is a smallest object of $Y$, i.e., for any object $z \in Y$ there is a morphism $y \rightarrow z$.

(ii) $e$ equalizes any two morphisms with the same codomain and domain $y$, i.e., any diagram $y \rightarrow \dot{\rightarrow} \mathrm{y}_{\rightarrow} z$ is commutative.

At present, I can prove the equivalence of (1) and (2) and the implication (3) $\rightarrow(1)$ in general, i.e., without any additional condition on $X$. The implication $(1) \rightarrow(3)$ holds at least if one of the following conditions on $X$ is satisfied: 